\title{
CIRCULAR CORRELATION COEFFICIENTS VERSUS THE PHASE-LOCKING-VALUE
}

\author{
Katrin Pauen and Galina Ivanova \\ Departement of Computer Science, Humboldt-Universität zu Berlin, Germany \\ katrin.pauen@informatik.hu-berlin.de
}

\begin{abstract}
The phase-locking-value is one of the most popular measures to quantify bivariate phase coupling. Recently, the use of more complex circular statistical methods for the quantification of synchronization has been discussed. Motivated by these new developments, the PLV is compared with a simple and a multiple circular-circular correlation coefficient. In the process, the reliability of the three measurements is tested using simulated signals and their applicability to real EEG data is demonstrated. Finally, advantages and disadvantages of the methods are discussed.
\end{abstract}

Keywords: Circular-circular correlation, EEG, synchronization, phase coupling, phase-locking-value

\section{Introduction}

The synchronization of different physiological processes represents an important issue in many biomedical research fields. Coupling may not only occur within one physiological system, as for instance the brain, but also between distinct systems, as heartbeat and respiration show [6]. Thus, the quantification of such coupling is a crucial objective in biomedical signal analyses and a lot of coupling measures have already been introduced [1]. However, many of these measurements mix amplitude and phase information and cannot quantify phase synchronization regardless of existing amplitude dependencies. To capture pure phase relationships, the instantaneous phases of the signals have to be considered. The most popular measurement using this approach is certainly given by the phase-locking-value PLV [3]. This measure calculates bivariate phase differences and quantifies rotations and reflexions of the circular phase variables. Recently, the benefit of more complex circular statistical methods for the quantification of phase associations has been discussed and two multiple circular-circular correlation coefficients have been presented [4]. But can these more complex coefficients keep pace with the oldestablished phase-locking-value? This question will be addressed in the present paper.

\section{Methods}

In the following, the PLV will be compared with the most promising multiple circular-circular correlation coefficient, namely the coefficient according to Jupp and Mardia $r_{m, J M}^{2}$ [4], and its simple counterpart $r_{s, J M}^{2}$ [2]. In the process, the two most important properties of a reliable coupling measure will be investigated. Firstly, it should take values close to one if synchronous or phase-coupled signals are considered. Secondly, the values should draw near zero if asynchronous signals are examined. To study these characteristics in detail, a comparable set of synchronous and asynchronous signals is required. To this end, 100 asynchronous oscillators $s_{k}$ are simulated each consisting of 15 added cosines with integer frequencies $f_{i} \sim U(1,30)$ and belonging amplitudes $A_{i} \sim N\left(20 / f_{i}, 2^{2}\right)$. Subsequently, various phase-coupled signals $s_{k}^{(l)}$ are obtained to each oscillator $s_{k}$ by inserting a pseudorandom phase shift $\alpha_{l} \sim U(-\pi, \pi)$. An example for two such coupled oscillators $s_{1}^{(1)}$ and $s_{1}^{(2)}$ and an according asynchronous signal $s_{2}^{(1)}$ is illustrated in fig. 1 . To simulate various realizations of the same process,

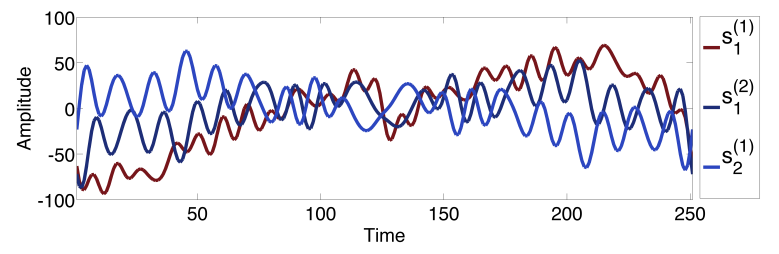

Figure 1: Exemplary illustration of the simulated signals.

it is assumed that the amplitudes and frequencies of an oscillator remain stable, while its zero phases vary slightly from trial to trial following a von Mises distribution with $\varphi_{0} \sim M(\mu, 1)$ and $\mu \sim U(-\pi, \pi)$. Finally, a normally distributed phase noise $w(j) \sim N(0,0.12)$ is added to the zero phases of the oscillators and they are superimposed with some gaussian noise $n(j, t) \sim N(0,52)$ since real biomedical signals are commonly noisy. To estimate the instantaneous phases of the signals, the Hilbert transform is utilized.

\section{Results}

Obviously, a comparison of simple and multiple synchronization measures is only partly possible. Whereas the PLV and the simple coefficient can only take into account two signals, the multiple coefficient requires at least three oscillators of which two function as predictors. To assure maximal comparability, this additional second predictor is always chosen to be phase-locked to the first one. Unlike the circular correlation coefficients, the PLV may only be utilized in multi trial analyses. Thus, the performance of all measures can only be compared in this kind of examination in which the extent of synchronization is estimated for every point in time. As the synchrony, or asynchrony, between the oscillators remains stable over time, these estimates can then be averaged. Tab. 1 shows the mean results for 100 ex- 
aminations as well as the belonging percentages of significant tests. These tests are chosen according to the different measurements and are performed with a significance level of 0.01. Obviously, the PLV exhibits the highest values

\begin{tabular}{lllll}
\multicolumn{3}{c}{ Table 1: Results for simulated data. } \\
\hline measure & \multicolumn{2}{c}{ synchronous } & \multicolumn{3}{c}{ asynchronous } \\
& value & sig. tests & value & sig. tests \\
\hline PLV & 0.8985 & $98.71 \%$ & 0.1848 & $33.33 \%$ \\
$r_{s, J M}^{2}$ & 0.8082 & $98.16 \%$ & 0.0205 & $01.46 \%$ \\
$r_{m, J M}^{2}$ & 0.8436 & $98.45 \%$ & 0.0413 & $01.26 \%$ \\
\hline
\end{tabular}

together with the greatest proportion of significant tests in both conditions. Furthermore, the multiple correlations are higher than their simple counterparts. Regardless of these minor differences, all measurements provide good results for truly phase-coupled signals: at least $98.16 \%$ of the tests carried out indicate a dependency. Thus, the first quality criteria, values close to one for synchronous signals, is fulfilled by all measures. Unfortunately, these good outcomes cannot be replicated for asynchronous signals. Although all values remain below $0.1848,33.33 \%$ of the phase-lockingtests become falsely positive. In contrast, the percentage of wrongly significant tests for the circular coefficients does not exceed $1.46 \%$ and is even lower for the multiple coefficient. Hence, the extent of phase-coupling may be overestimated with the phase-locking-value. The same pattern,

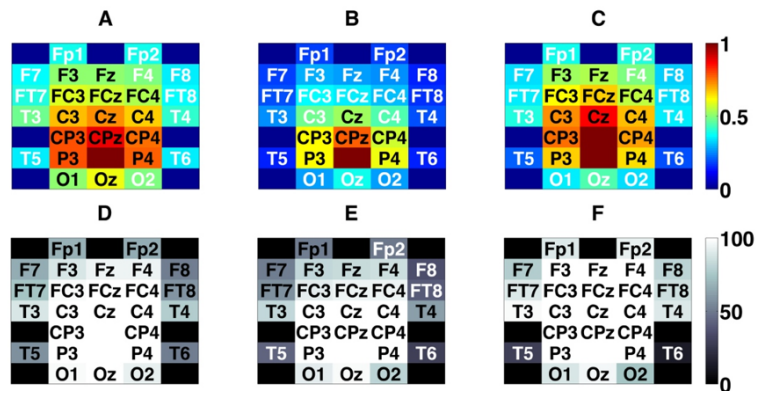

Figure 2: Estimated theta couplings (A-C) and according percentages of significant tests (D-F). A: PLV, B: $r_{J M, s}^{2}, \mathbf{C}$ : $r_{J M, m}^{2}, \mathbf{D}:$ PLV-tests, E: $r_{J M, s}^{2}$-tests, $\mathbf{F}: r_{J M, m}^{2}$-tests.

comparable high overall values for the PLV, is observable in an exemplary analysis of real EEG data. Here, a randomly selected data set of an acoustic oddball experiment is chosen that consists of 40 trials and 28 electrodes with mastoids as reference. In accordance with current research, induced theta oscillations following a P300 are examined and fronto-central couplings are expected [5]. To this end, signals are initially preprocessed in an appropriate manner and theta is filtered out using a butterwort filter of passband 4-8 hertz. Subsequently, the instantaneous signal phases are extracted using the Hilbert transform. As midline couplings are expected, electrode $\mathrm{Pz}$, and for the multiple coefficient additionally $\mathrm{CPz}$, is chosen as predictive variable and the extent of synchronization with every other electrode is estimated. This is done within a time segment from 500 to 3000 ms after target presentation in multi trial analyses. Fig. 2 A shows the time-averaged results for the PLV, while picture B illustrates the outcomes for the simple circular-circular correlation coefficient and picture $\mathbf{C}$ the according values for the multiple one. In addition, illustration $\mathbf{D}-\mathbf{F}$ depict the corresponding percentages of significant tests that all capture the suspected fronto-central couplings. In contrast, only little up to low correlations with electrodes $\mathrm{FCz}$ (0.3461) and $\mathrm{Fz}(0.2764)$ can be achieved with the simple coefficient. However, the multiple correlations with these electrodes (FCz: 0.6657; Fz: 0.5460) are even higher than the corresponding PLVs (FCz: 0.5749; Fz: 0.5060) along with more significant test results (FCz: 100\%; Fz: 100\% versus FCz: 94.72\%; Fz: 98.56\%).

\section{Discussion}

In total, circular-circular correlation coefficients are well suited to estimate phase-coupling in a reliable manner. Although they take slightly lower values than the PLV together with less significant test results for simulated data, they can keep pace with this traditional measure. Unlike the PLV, they are even able to distinguish between truly phase-coupled and just individually phase-locked signals. Furthermore, they are not restricted to bivariate analyses and can include an arbitrary number of predictive variables. As the circular coefficients may also be utilized in single trial analyses, a further evaluation of this new circular approach seems worthwhile. In the process, particular attention should be paid to the multiple coefficient since this measure exhibited even higher midline coupling estimates than the PLV.

\section{Bibliography}

[1] K. J. Blinowska, "Review of the methods of determination of directed connectivity from multichannel data," Med. Biol. Eng. Comput., vol. 49, pp. 521-529, 2011.

[2] P. E. Jupp and K. V. Mardia, ”A general correlation coefficient for directional data and related regression problems", Biometrika, vol. 67, pp. 163-173, 1980.

[3] J.-P. Lachaux, E. Rodriguez, J. Martinerie and F. J. Varela, "Measuring Phase Synchrony in Brain Signals," Human Brain Mapping, vol. 8, pp. 194-208, 1999.

[4] K. Pauen and G. Ivanova, "Multiple CircularCircular Correlation Coefficients for the Quantification of Phase Synchronization Processes in the Brain," Biomedical Engineering, vol. 0, pp. 1-15, 2013.

[5] J. Yordanova and V. Kolev, "Single-sweep analysis of the theta frequency band during an auditory oddball task," Psychophysiology, vol. 35, pp. 116-126, 1998.

[6] C. Schäfer, M. G. Rosenblum, H.-H. Abel and J. Kurths, "Synchronization in the human cardiorespiratory system," Physical Review E., vol. 60, pp. 857870 , July 1999. 